\title{
Low-Temperature Deposition of Highly Conductive Aluminum Metal Films on Flexible Substrates Using Liquid Alane MOD Precursors
}

\author{
Samuel P. Douglas and Caroline E. Knapp*
}

Cite This: ACS Appl. Mater. Interfaces 2020, 12, 26193-26199

Read Online

ABSTRACT: Metal-organic decomposition (MOD) precursor inks are emerging as the new route to low-temperature deposition of highly conductive metals, owing to the tunability of their decomposition. New methods of printing are being investigated to help negate the progressive issues of the electronics industry, not least the movement toward low-cost polymers and paper substrates. Informed precursor design is crucial if achieving materials capable of this is possible. In this work, the liquid MOD precursors, dimethylethylamine alane (DMEAA) and trimethylamine alane (TEAA), have been used to deposit a highly conductive aluminum $(\mathrm{Al})$ metal with resistivities in the range of $4.10 \times 10^{-5}$ to $5.32 \times 10^{-7} \Omega \mathrm{m}$ (mean electrical resistivity of $8 \times$

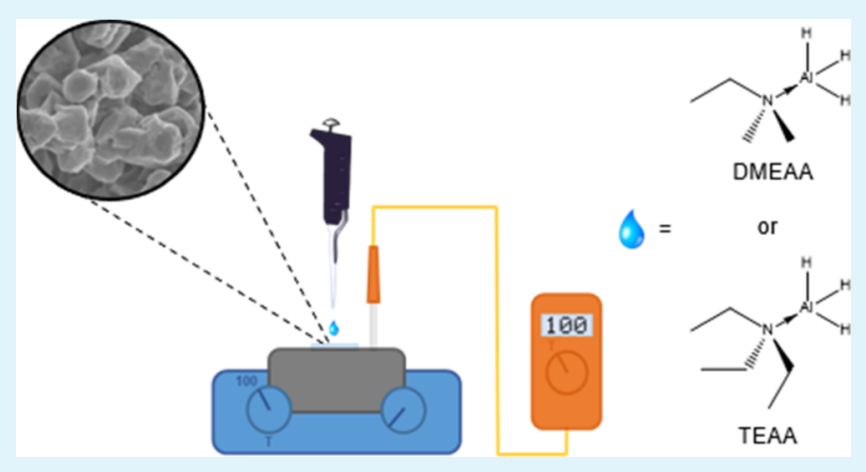
$10^{-6} \Omega \mathrm{m}$, approximately 300 times more resistive than bulk $\mathrm{Al}$ metal), without the need for an additional solvent, at low temperatures $\left(100\right.$ and $\left.120{ }^{\circ} \mathrm{C}\right)$, on a range of substrates including glass, polyimide, polyethylene terephthalate, and paper. Conductive coatings have been analyzed using X-ray diffraction, scanning electron microscopy, energy-dispersive X-ray spectroscopy, X-ray photoelectron spectroscopy and resistivity measurements; as a proof of concept, $\mathrm{Al}$ deposited on paper has been used in an electrical circuit. Results indicate that DMEAA is a better precursor, producing more conductive films, which is explained by its lower decomposition temperature and higher $\mathrm{Al}$ weight loading, indicating potential for significant industrial application.

KEYWORDS: aluminium precursors, MOD inks, metal deposition, low temperature, flexible substrates, conductive films

\section{INTRODUCTION}

As the world's technological demands increase, there is a push for device fabrication to become more material, energy and time efficient. Hence, materials chemists must adapt precursor design $^{1}$ to be better compatible with modern lower temperature deposition techniques, such as printing: a method of device fabrication that satisfies these objectives. To reap the benefits of using printing techniques for device fabrication, designer inks which transform at low temperatures (affording compatibility with low-cost flexible substrates) are needed. One class of designer ink that can be used to deposit highly conductive metal coatings is metal-organic decomposition (MOD) precursor inks. ${ }^{2}$ These inks contain a metal-organic precursor in solution, and upon some form of initiation, such as thermal ${ }^{3,4}$ or plasma sintering, ${ }^{5,6}$ the compound decomposes to the metallic element through reduction of the metal center, along with removal of ligands as gaseous byproducts. An ideal MOD precursor should produce coatings with a conductivity comparable to the bulk metal, have a reasonable shelf life, be able to undergo low-temperature processing $\left(<200{ }^{\circ} \mathrm{C}\right)$, and require short treating times. These conditions should be satisfied in order to deposit onto low-cost flexible substrates such as paper/plastic via fast-moving industrial process lines. It is common for MOD complexes to require an inert atmosphere to prevent oxidation ${ }^{7}$ or a reducing atmosphere (e.g., $\mathrm{H}_{2} /$ formic acid vapor/titanium isopropoxide vapor) ${ }^{8-10}$ to aid conversion to the metal.

Aluminum ( $\mathrm{Al})$, being the fourth least resistant metal $(2.65$ $\left.\times 10^{-8} \Omega \mathrm{m}, 20^{\circ} \mathrm{C}\right)^{11}$ and the most abundant metallic element in the Earth's crust, ${ }^{12}$ is a popular candidate for use in MOD inks, in order to form highly electrically conductive metallic features. In addition to high conductivity, Al's very low work function makes it an excellent material to make electrodes for ohmic contact. ${ }^{10}$ However, $\mathrm{Al}$ is highly prone to oxidation to metal oxides and hydroxides, which exhibit high electrical resistivities, which is why $\mathrm{Al}$ MOD precursors historically have been less extensively researched than their silver, copper, or gold counterparts. ${ }^{2}$ A solution to this problem is to deposit and print $\mathrm{Al}$ under vacuum, though this has high operating outlays

Received: March 23, 2020

Accepted: May 18, 2020

Published: May 18, 2020 
and has limitations in pattern printing on fibrous substrates such as fabric or paper. ${ }^{13}$

Until the mid-1990's, chemical vapor deposition (CVD) of $\mathrm{Al}$ used $\mathrm{Al}$ alkyls because of commercial availability. Triisobutylaluminum received attention as an $\mathrm{Al}$ CVD precursor; however, high decomposition temperatures in excess of $500{ }^{\circ} \mathrm{C}$ are required to break the $\mathrm{Al}-\mathrm{C}$ bond. ${ }^{14-16}$ An alternative approach is to use compounds containing the $\mathrm{Al}-\mathrm{H}$ bond, namely aluminum trihydride compounds, which are generally more volatile and less air-sensitive. ${ }^{17}$ Aluminum trihydride, $\mathrm{AlH}_{3}$ or alane, itself displays a low decomposition temperature $\left(>60^{\circ} \mathrm{C}\right)$ and decomposes cleanly to produce $\mathrm{Al}$ and $\mathrm{H}_{2}{ }^{18}$ Unfortunately, alane polymerizes to form an insoluble solid, making it unable to be used in solution in the form of a MOD ink. By complexing alane with a donor atom, commonly oxygen or nitrogen, a full octet of valence electrons around the $\mathrm{Al}$ center is achieved, which renders the alane complex stable for polymerization, while retaining a low decomposition temperature. ${ }^{15}$

The preparation and properties of such Lewis base stabilized alane compounds have been known for some time. ${ }^{19}$ The first example of use of such compounds to form $\mathrm{Al}$ thin films from the vapor phase was in the late 1960's patent literature. ${ }^{15}$ Glatfelter and co-workers ${ }^{17}$ were the first to publish such work outside the patent literature two decades later, with several contributions to the field of $\mathrm{Al}$ metal CVD. It has only been more recently that these types of compounds have been applied to solution-based processing procedures under an inert atmosphere, again with many only featured in the patent literature, due to their highly industrially exciting applications. $^{20,21}$

Lee and coworkers have significantly contributed to the literature of Al-based MOD inks. In 2011, ${ }^{10}$ they proposed a novel warm solution-stamping process to fabricate highly conductive $\mathrm{Al}$ deposits, using $n$-butylether stabilized alane, $\left[\mathrm{AlH}_{3} \cdot\left\{\mathrm{O}\left(\mathrm{C}_{4} \mathrm{H}_{9}\right)_{2}\right\}\right]$. The films produced displayed excellent electrical properties on rigid and flexible substrates, with a minimum electrical resistivity of $8 \times 10^{-8} \Omega \mathrm{m}$, three times higher resistivity than that of bulk metal, which was achieved at a sintering temperature of $150{ }^{\circ} \mathrm{C}$, along with promising mechanical properties. The precursor decomposed in a twostep process, with the aid of evaporated titanium isopropoxide, $\left[\mathrm{Ti}\left(\mathrm{O}^{i} \mathrm{Pr}\right)_{4}\right]$ (under an inert atmosphere) to aid reduction and further prevent oxidation of $\mathrm{Al}$ deposits. This reactive species aided thermal sintering became a popular methodology in the Lee group; however, descriptions of how the $\mathrm{Ti}$ catalyst is recovered/separated are not clear. Experiments without a catalyst are reported to thermally sinter at $165^{\circ} \mathrm{C}$. Lee Led $^{22}$ used the same reactive sintering process in 2012 to deposit $\mathrm{Al}$ films of $c a .50 \mu \mathrm{m}$ thickness onto numerous types of paper-the best deposits coated inkjet printing paper with a lowest resistivity of ca. $6 \times 10^{-7} \Omega \mathrm{m}$ recorded, which is approximately 23 times that of the bulk metal. In 2013, Lee et al. ${ }^{23}$ employed a solution-dipping process, again using $\left[\mathrm{AlH}_{3} \cdot\left\{\mathrm{O}\left(\mathrm{C}_{4} \mathrm{H}_{9}\right)_{2}\right\}\right]$, which allowed the preparation of larger area substrates. Lee dipped a preheated $\left(100{ }^{\circ} \mathrm{C}\right)$ and pretreated $\left(\left[\mathrm{Ti}\left(\mathrm{O}^{i} \mathrm{Pr}\right)_{4}\right]\right)$ substrate into the precursor solution at room temperature. In 2018, Lee's group ${ }^{24}$ took major steps in industrializing the use of their $\left[\mathrm{AlH}_{3} \cdot\left\{\mathrm{O}\left(\mathrm{C}_{4} \mathrm{H}_{9}\right)_{2}\right\}\right]$ precursor, by applying it to an inert atmosphere roll-to-roll process to print $\mathrm{Al}$ thin films on polyimide (PI) using a diluted catalyst (100:1 volumetric ratio of dibutyl ether to [Ti$\left.\left.\left(\mathrm{O}^{i} \mathrm{Pr}\right)_{4}\right)\right]$. Films exhibited high durability and good adhesion, with a best resistivity of $1.70 \times 10^{-7} \Omega \mathrm{m}$ for a film that was $60.9 \mathrm{~nm}$ thick, which is six times more resistant than the bulk metal.

$\left[\mathrm{AlH}_{3} \cdot\left\{\mathrm{O}\left(\mathrm{C}_{4} \mathrm{H}_{9}\right)_{2}\right\}\right]$ is not stable over time and as such $\mathrm{Lee}^{25}$ in 2014 reported the innovative solution of producing an ink which could be produced by redissolving a powder-type alane composite. Amines are stronger Lewis bases than ethers, so they form more stable donor acceptor bonds to the alane moiety, and hence, trimethylamine alane (TMAA), $\left[\mathrm{AlH}_{3}\right.$. $\mathrm{NMe}_{3}$ ] was used. TMAA was dissolved in various solvents and films were printed using a solution-stamping process (substrate at $130{ }^{\circ} \mathrm{C}$ ) under an inert atmosphere. The films produced displayed excellent electrical properties on both glass and polyethylene terephthalate (PET) substrates. All the $\mathrm{Al}$ deposits on glass substrates with the as-prepared and 180day-stored precursor powder show electrical resistivity levels of 5.8 to $7.0 \times 10^{-8} \Omega \mathrm{m}$, a minimum of just over twice as resistive as the bulk metal. Shen ${ }^{26}$ had previously used a solution process using triethylamine alane (TEAA), $\left[\mathrm{AlH}_{3}\right.$. $\left.\mathrm{NEt}_{3}\right]$ as a precursor; however, the process was expensive ( $\mathrm{Pt}$ catalyst required), required a high boiling point amine as the solvent, and produced films of high surface roughness.

Herein, we exploit the reactivity of amine-stabilized alanes that are liquids at room temperature and can be used without further dilution or formulation. We report the use of these liquid amine-stabilized alane MOD inks to deposit a highly conductive $\mathrm{Al}$ metal $\left(4.10 \times 10^{-5}\right.$ to $\left.5.32 \times 10^{-7} \Omega \mathrm{m}\right)$, without the need for any additives or catalysts at low temperatures $\left(100,120^{\circ} \mathrm{C}\right)$, on a range of substrates including glass, PI, PET, and paper.

\section{EXPERIMENTAL SECTION}

Materials. All reagents were procured from Sigma-Aldrich/Merck unless stated otherwise. All reactions were performed using standard Schlenk and glove box techniques in vacuo or under an atmosphere of $\mathrm{N}_{2}$. All nondeuterated solvents were predried using dry solvent stills and were further dried over the appropriately sized molecular sieves (3 or $4 \AA$ ). Deuterated solvents were obtained from Cambridge Isotope Laboratories, which were degassed and stored over $4 \AA$ molecular sieves. Both dimethylethylamine (DMEA) and triethylamine (TEA) were distilled in vacuo and were kept in a $7{ }^{\circ} \mathrm{C}$ fridge. $\mathrm{AlCl}_{3}$ anhydrous powder, sublimed for synthesis, $\geq 98 \%$ was used as received, and $\mathrm{LiAlH}_{4}$ powder, reagent grade, $95 \%$ was purified by dissolution in and recrystallization from $\mathrm{Et}_{2} \mathrm{O}$ using a literature procedure. ${ }^{27}$ DMEAA and TEAA complexes were stored in vials in a glove box fridge prior to use. Al metal films were deposited onto preheated glass, PI, PET, and paper (Fujifilm Instax) substrates with thicknesses of $1.00,1.06,1.05$, and $0.85 \mathrm{~mm}$, respectively.

Synthesis of Dimethylethylamine Alane, $\left[\mathrm{AlH}_{3} \cdot \mathrm{NMe}{ }_{2} \mathrm{Et}\right]$ (DMEAA). A slurry of $\mathrm{AlCl}_{3}(1.0642 \mathrm{~g}, 7.98 \mathrm{mmol})$ in hexane (ca. $20 \mathrm{~mL}$ ) was added to a slurry of $\mathrm{LiAlH}_{4}(0.9999 \mathrm{~g}, 26.4 \mathrm{mmol})$ in hexane $($ ca. $20 \mathrm{~mL})$ at $-78{ }^{\circ} \mathrm{C}$, which was stirred for $30 \mathrm{~min}$. Following this, DMEA $(3.8 \mathrm{~mL}, 35.1 \mathrm{mmol})$ was added at $-78^{\circ} \mathrm{C}$. The reaction was stirred under cooling for $30 \mathrm{~min}$, before being left to stir overnight while warming to room temperature. The resultant cloudy pale grey mixture was filtered to produce a transparent colorless solution, which was concentrated by $c a$. half before being cooled to $-78{ }^{\circ} \mathrm{C}$ to form a white solid in a transparent colorless solution. The solid was separated from the solution by cannula filtration under cooling. The solid was redissolved with new hexane (ca. $20 \mathrm{~mL}$ ) and warmed to room temperature, before being separated at $-78{ }^{\circ} \mathrm{C}$ as before. Finally, the white solid was dried in vacuo under cooling $\left(<-10{ }^{\circ} \mathrm{C}\right)$ to remove any excess hexane, to produce a colorless transparent liquid at room temperature (yield: $2.16 \mathrm{~g}$, 65.6\%). ${ }^{1} \mathrm{H}$ NMR $\left(500 \mathrm{MHz}, \mathrm{C}_{6} \mathrm{D}_{6}\right): \delta 3.84\left(\mathrm{br}, 3 \mathrm{H}, \mathrm{Al}-\underline{H}_{3}, \mathrm{fwhm}=\right.$ $18.83 \mathrm{MHz}$ ), $2.38\left(\mathrm{q}, J=7.3 \mathrm{~Hz}, 2 \mathrm{H}, \mathrm{N}-\underline{\mathrm{C}}_{2}\right), 2.00\left(\mathrm{~s}, 6 \mathrm{H}, \mathrm{N}-\mathrm{C}_{3}\right)$, 


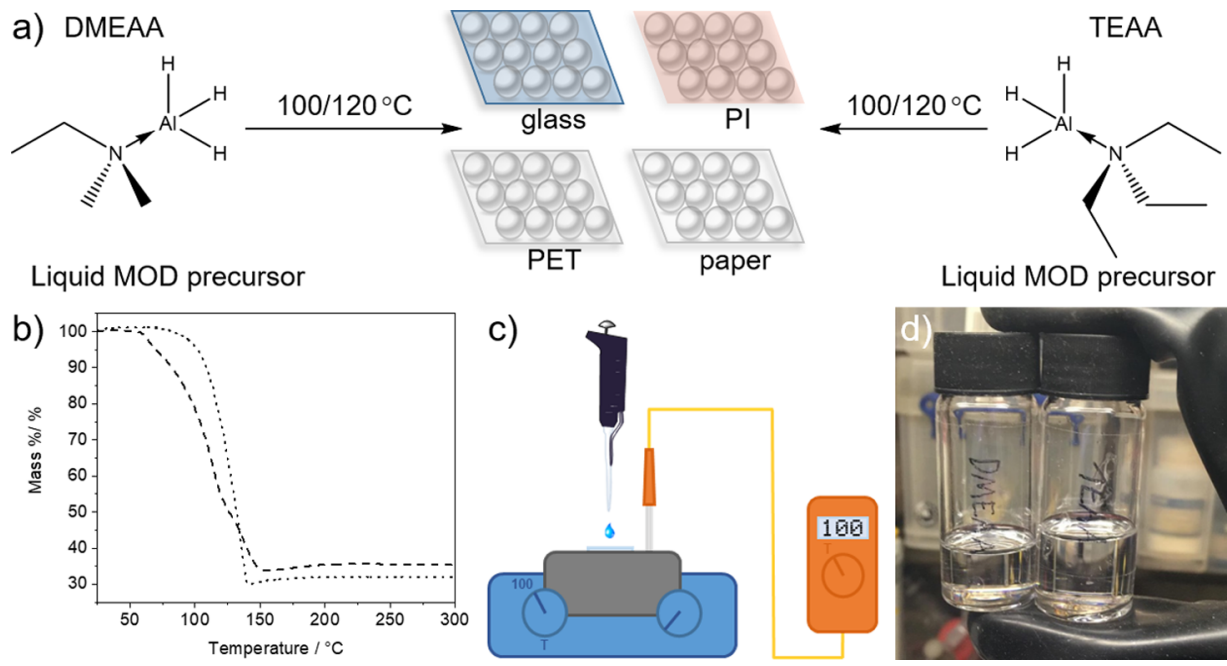

Figure 1. Deposition of liquid MOD inks onto glass, PI, PET, and paper. (a) Schematic of the process, including chemical structures of the liquid MOD precursors (b) TGA profiles of DMEAA (dotted line) and TEAA (dashed line). (c) Schematic of the deposition process. (d) Photograph of the two-liquid MOD inks.

$0.83\left(\mathrm{t}, J=7.3 \mathrm{~Hz}, 3 \mathrm{H}, \mathrm{CH}_{3}\right) \cdot{ }^{13} \mathrm{C} \mathrm{NMR}\left(126 \mathrm{MHz}, \mathrm{C}_{6} \mathrm{D}_{6}\right): \delta 53.60$ $\left(\mathrm{N}-\mathrm{CH}_{2}\right), 44.06\left(\mathrm{~N}-\mathrm{CH}_{3}\right), 9.11\left(\mathrm{CH}_{2} \mathrm{CH}_{3}\right)$. NMR data are in agreement with the literature. ${ }^{28}$

Synthesis of Triethylamine Alane, $\left[\mathrm{AlH}_{3} \cdot \mathrm{NEt}_{3}\right]$ (TEAA). A slurry of $\mathrm{AlCl}_{3}(1.0667 \mathrm{~g}, 8.00 \mathrm{mmol})$ in hexane $(c a .20 \mathrm{~mL})$ was added to a slurry of $\mathrm{LiAlH}_{4}(1.0010 \mathrm{~g}, 24.5 \mathrm{mmol})$ in hexane (ca. 20 $\mathrm{mL}$ ) at $-78{ }^{\circ} \mathrm{C}$, which was stirred for $30 \mathrm{~min}$. Following this, TEA $(4.9 \mathrm{~mL}, 35.2 \mathrm{mmol})$ was added at $-78{ }^{\circ} \mathrm{C}$. The reaction mixture was stirred under cooling for $30 \mathrm{~min}$, before being left to stir overnight while warming to room temperature. The resultant cloudy pale grey mixture was filtered to produce a transparent colorless solution, which was concentrated by $\mathrm{ca}$. half before being cooled to $-78^{\circ} \mathrm{C}$ to form a white solid in a transparent colorless solution. The solid was separated from the solution by cannula filtration under cooling. The solid was re-dissolved with new hexane (ca. $20 \mathrm{~mL}$ ) and warmed to room temperature, before being separated at $-78{ }^{\circ} \mathrm{C}$ as before. Finally, the white solid was dried in vacuo under cooling $\left(<-10{ }^{\circ} \mathrm{C}\right)$ to remove any excess hexane, to produce a colorless transparent liquid at room temperature (yield: $3.63 \mathrm{~g}, 87.2 \%) .{ }^{1} \mathrm{H}$ NMR $\left(500 \mathrm{MHz}, \mathrm{C}_{6} \mathrm{D}_{6}\right): \delta$ $4.09\left(\mathrm{br}, 3 \mathrm{H}, \mathrm{Al}-\underline{H}_{3}, \mathrm{fwhm}=190.73\right), 2.35(\mathrm{q}, J=7.3 \mathrm{~Hz}, 6 \mathrm{H}, \mathrm{N}-$ $\left.\mathrm{C}_{2}\right), 0.85\left(\mathrm{t}, J=7.3 \mathrm{~Hz}, 9 \mathrm{H}, \mathrm{CH}_{2} \mathrm{C}_{3}\right) .{ }^{13} \mathrm{C}$ NMR $(126 \mathrm{MHz}$, $\left.\mathrm{C}_{6} \mathrm{D}_{6}\right): \delta 48.21\left(\mathrm{~N}-\underline{C H}_{2}\right), 9.05\left(\mathrm{CH}_{2} \mathrm{CH}_{3}\right) . \mathrm{NMR}$ data are in agreement with the literature. ${ }^{29}$

Preparation of Al Metal Depositions. Al metal thin films were deposited on numerous substrates (glass, PI, PET, and paper) by a drop-casting process. Then, $25 \mu \mathrm{L}$ of neat liquid compound was dropped onto a preheated substrate $\left( \pm 1{ }^{\circ} \mathrm{C}\right.$ error in the temperature measurement of the hot surface) under an $\mathrm{N}_{2}$ atmosphere inside a glove box for a fixed time period. Films were sintered using a hotplate, using a Kewtech KT116 multimeter as an external temperature control.

Preparation of Circuit. A closed series circuit was constructed from a $9 \mathrm{~V}$ battery, a $10 \mathrm{k} \Omega$ resistor [to prevent light-emitting diode (LED) burn out], and a red $5 \mathrm{~mm}$ dual in-line package LED (fitted with a $12 \mathrm{~V}$ internal resistor).

Characterization (and Evaluation). NMR: ${ }^{1} \mathrm{H}$ and ${ }^{13} \mathrm{C}$ NMRs were collected on a Bruker $500 \mathrm{MHz}$ NMR instrument at $298 \mathrm{~K} .{ }^{1} \mathrm{H}$ NMRs were calibrated using the $\mathrm{C}_{6} \mathrm{D}_{6}$ singlet peak at $7.16 \mathrm{ppm}$, and ${ }^{13} \mathrm{C}$ NMRs were calibrated using the $\mathrm{C}_{6} \mathrm{D}_{6}$ triplet peak at $128.06 \mathrm{ppm}$. Thermogravimetric analysis (TGA): measurements were made using a Netzsch STA 449C Jupiter instrument, which had a sensitivity of 0.1 mg. Samples were placed in $85 \mu \mathrm{L} \mathrm{Al}$ pans that were sealed in a glove box and were pierced just before measurement to allow for mass loss. The samples were heated from $<30{ }^{\circ} \mathrm{C}$ up to a maximum of $300{ }^{\circ} \mathrm{C}$, at a heating rate of $10{ }^{\circ} \mathrm{C} \mathrm{min}{ }^{-1}$ under the flow of $\mathrm{He}$ shield gas. Electrical resistance measurements: lowest resistance measurements were recorded using a Kewtech KT116 multimeter. Resistivities were calculated with these values for the thicknesses of films produced; full details of calculating the values are given in the Supporting Information. Grazing incidence X-ray diffraction (XRD): patterns were measured using a Bruker-Axs diffractometer with parallel beam optics, fitted, with a PSD LynxEye silicon strip detector. X-rays were generated using a copper source with $\mathrm{Cu}_{\alpha 1}(\lambda=1.54056 \AA)$ and $\mathrm{Cu}$ $\mathrm{K}_{\alpha 2}(\lambda=1.54439 \AA)$ radiation with an intensity ratio of $2: 1$. The incident beam angle, $\theta$, was kept at $1^{\circ}$, with the angular range of scans collected between $20^{\circ}<2 \theta<66^{\circ}$. Data were collected using a step size of $0.05^{\circ}$ counted between 0.5 and $2 \mathrm{~s}$ per step. X-ray photoelectron spectroscopy (XPS): analysis of the films was carried out using a Thermo Scientific K-Alpha spectrometer equipped with a monochromatic $\mathrm{Al} \mathrm{K}_{\alpha}$ source in order to identify constituent elements, their speciation and atomic\%. The peaks were calibrated to the adventitious carbon $1 \mathrm{~s}$ standard peak $(284.8 \mathrm{eV})$ using CasaXPS, in order to compensate for charging of the surface. Survey scans were collected in the range of $0-1350 \mathrm{eV}$ (binding energy) at a pass energy of $40 \mathrm{eV}$ and were used to estimate atomic \%. C 1s, N 1s, $\mathrm{O} 1 \mathrm{~s}$, and $\mathrm{Al} 2 \mathrm{p}$ regions were scanned at the surface and after a $300 \mathrm{~s}$ etch, with each scan lasting $20 \mathrm{~s}$. Etching was achieved using an Ar ion beam with a voltage of $1 \mathrm{keV}$ and current of $1.55 \mu \mathrm{A}$. Scanning electron microscopy (SEM): samples were sputtered with a fine layer of gold. Images were collected by JEOL JSM-6301F field emission SEM at an accelerating voltage of $5 \mathrm{kV}$. Energy-dispersive X-ray spectroscopy (EDS) was measured with an acceleration voltage of 20 $\mathrm{kV}$.

\section{RESULTS AND DISCUSSION}

The patterning of molten metals is incompatible with affordable flexible substrates that are highly desired in the printing of electronic materials in the present day. This mismatch is due in part to the high melting point of metals (e.g., $\mathrm{Al}=660^{\circ} \mathrm{C}$ ) and the glass transition temperature of a range of plastic substrates being considerably lower or the deformation temperature of paper $\left(c a .100{ }^{\circ} \mathrm{C}\right)$. An obvious solution to this problem is the use of either nanoparticle (NP) inks to print metals (limited to silver, copper, gold industrially $)^{30}$ or precursors in solutions, also known as MOD inks. One criticism of MOD inks is that the weight loading of the metal in these solutions is comparatively low when compared to a NP equivalent. In the work presented here, liquid Al precursors have been used as MOD inks, with no further dilution, resulting in higher weight loadings 

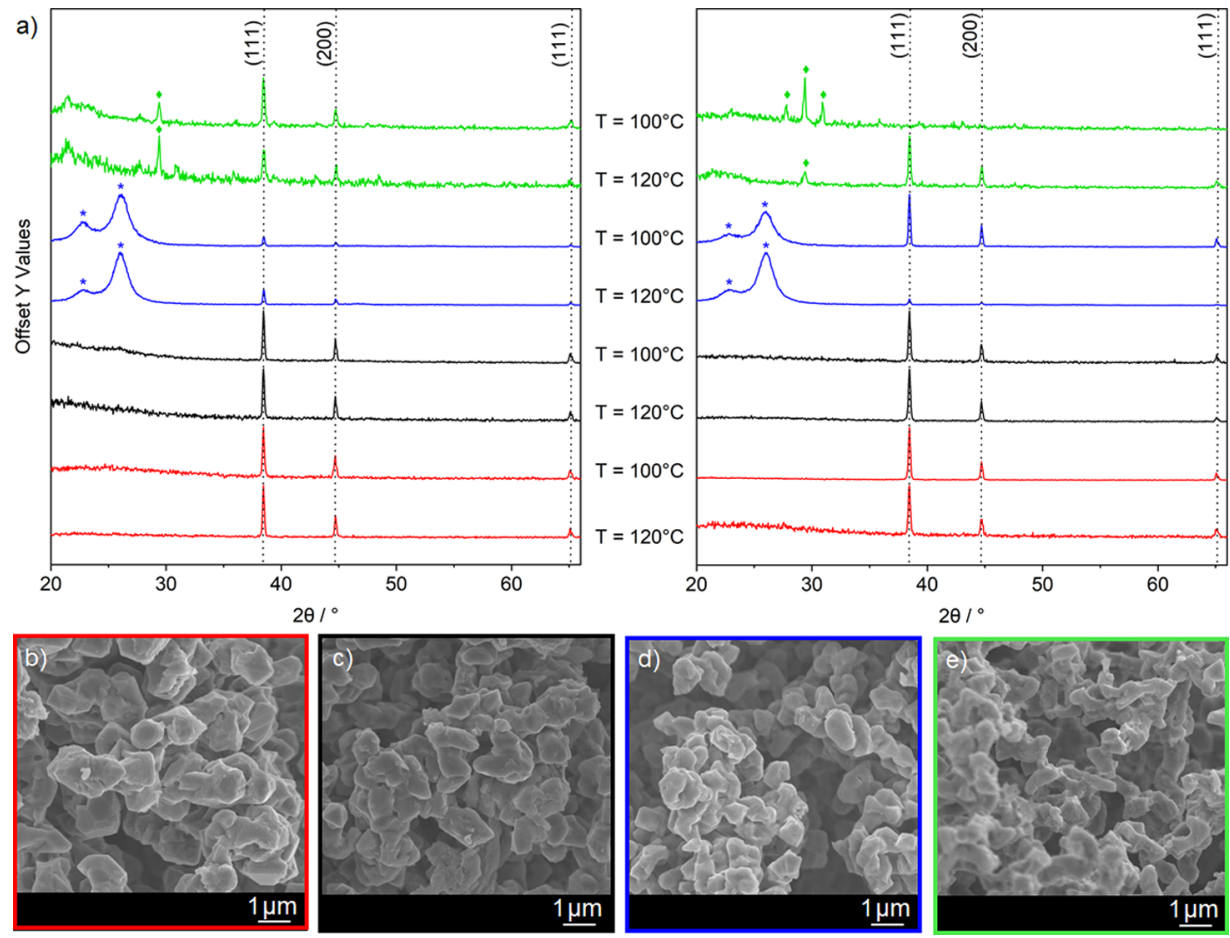

Figure 2. (a) Representative XRD patterns of films deposited using DMEAA (left) and TMEAA (right) on substrates: glass (red), PI (black), PET (blue), and paper (green). Sintering temperatures are given in the center, $*$ and $\diamond$ indicate PET and paper substrate peaks, respectively. (b-e) Representative top-down SEM images of films on glass, PI, PET, and paper.

$(\mathrm{DMEAA}=26.2 \% \mathrm{Al}$ by mole fraction, $\mathrm{TEAA}=20.6 \% \mathrm{Al}$ by mole fraction).

Highly conductive $\mathrm{Al}$ coatings on a range of substrates (glass, PI, PET, and paper) have been deposited at low temperatures $\left(100-120^{\circ} \mathrm{C}\right.$ ) using the amine stabilized alanes: DMEAA and TEAA under an atmosphere of nitrogen (Figure 1a). The expected products upon decomposition under $\mathrm{N}_{2}$ at elevated temperature (i.e. sintering conditions) are $\mathrm{Al}$ metal, hydrogen gas, and free amines, while in air at room temperature (i.e. general air-sensitive reactivity) aluminum oxide is formed in preference to metal (see Supporting Information for reaction schemes). Conductive $\mathrm{Al}$ coatings $\left(4.10 \times 10^{-5}\right.$ to $\left.5.32 \times 10^{-7} \Omega \mathrm{m}\right)$ comparable to the bulk metal have been achieved. To illustrate our approach that using alanes as precursors would considerably lower the temperature of deposition owing to the presence of $\mathrm{Al}-\mathrm{H}$ bonds, TGA was initially carried out of DMEAA and TEAA (Figure 1b).

Both complexes showed a clean single-step decomposition in TGA (Figure $1 \mathrm{~b}$ ), with the onset of decomposition beginning at 58 and $76{ }^{\circ} \mathrm{C}$, respectively; all decomposition is complete before $150{ }^{\circ} \mathrm{C}$. Mass losses of 70 and $66 \%$ are recorded; with mass losses of 74 and $79 \%$, expected if pure $\mathrm{Al}$ metal is formed, with discrepancies in values most likely due to the volatile nature of compounds. Dissociation of the amine is the ratedetermining step; hence why we observe different decomposition temperatures for the different precursors. A stronger donor-acceptor bond between the amine and alane moieties is formed in TEAA due to a greater amount of lone pair donation due to added hyperconjugation, as a result of having three ethyl groups compared to one in DMEAA, which is reflected by the $\mathrm{pK}$,'s of their conjugate acids (10.65 and 9.99, respectively). ${ }^{31}$ This is why we see TEAA decompose at a slightly higher temperature compared to DMEAA in the TGA profile. Decomposition of the $\mathrm{AlH}_{3}$ moiety is facile, with significant mass loss reported in the literature at $60{ }^{\circ} \mathrm{C}$, which is due to a combination of weak $\mathrm{Al}-\mathrm{H}$ bonds and the entropic driving force of $\mathrm{H}_{2}$ formation. ${ }^{18}$

Following TGA, both MOD inks DMEAA and TEAA were used to deposit highly conductive $\mathrm{Al}$ on a range of substrates including glass, PI, PET, and paper. A simple heating setup was used (Figure 1c), and both liquid MOD inks DMEAA and TEAA (Figure 1d) were used to deposit on all substrates at 100 and $120^{\circ} \mathrm{C}$; all films were uniform, and there was a good coverage of the substrate. Interestingly, attempts to deposit at $80{ }^{\circ} \mathrm{C}$ were unsuccessful despite long sintering times $(>20$ $\mathrm{min}$ ), while metallic features were observed, the films were not conductive.

In all cases, metallic coatings on the substrate were observed, and those on paper were well adhered to the substrate, however samples on PI, PET, and glass could be scratched from the surface with a steel stylus. XRD data were collected from all films in the range of $20^{\circ}<2 \theta<66^{\circ}$, which all contained patterns with three Bragg peaks at 38.5, 44.8, and $65.2^{\circ}$, which can be indexed to the Al metal (cubic space group: $F m \overline{3} m$ ) (Figure $2 \mathrm{a}$ ). Bragg peaks deriving from the substrate can also be observed in some patterns (denoted with $*$ and $\diamond$ ), with substrate and full (unnormalized) XRD data given in the Supporting Information.

Scanning electron microscopy (SEM) images were taken from a representative range of samples to study their surface morphology (Figure 2b-e). The morphology of the $\mathrm{Al}$ particles on glass, PI, and PET is very similar, showing spherical grains, with many coalesced together because of sintering. Average grain sizes of $1.43 \pm 0.09,1.08 \pm 0.05$, and $1.03 \pm 0.06 \mu \mathrm{m}$ were measured for glass, PI, and PET substrates, respectively (histograms in Supporting Information). The morphology of the $\mathrm{Al}$ film on paper is different to the other substrates, with a slightly more vermicular structure. 
Table 1. Resistivity Values for Depositions Using DMEAA and TEAA onto Various Substrates at 120 and $100{ }^{\circ} \mathrm{C}^{a}$

\begin{tabular}{|c|c|c|c|c|}
\hline precursor & substrate & temperature $/{ }^{\circ} \mathrm{C}$ & time $/ \mathrm{min} / \mathrm{s}$ & resistivity $/ \Omega \mathrm{m}$ \\
\hline \multirow[t]{8}{*}{ DMEAA } & glass & 100 & $1: 30$ & $8.29 \times 10^{-6} \pm 6.28 \times 10^{-7}$ \\
\hline & glass & 120 & 1:00 & $1.60 \times 10^{-6} \pm 4.21 \times 10^{-7}$ \\
\hline & PI & 100 & $1: 30$ & $6.38 \times 10^{-6} \pm 1.46 \times 10^{-6}$ \\
\hline & PI & 120 & 1:00 & $9.57 \times 10^{-7} \pm 2.33 \times 10^{-7}$ \\
\hline & PET & 100 & $2: 30$ & $1.91 \times 10^{-6} \pm 2.35 \times 10^{-7}$ \\
\hline & PET & 120 & $1: 30$ & $2.34 \times 10^{-6} \pm 4.86 \times 10^{-7}$ \\
\hline & paper & 100 & 2:00 & $2.98 \times 10^{-6} \pm 1.38 \times 10^{-6}$ \\
\hline & paper & 120 & $1: 30$ & $2.66 \times 10^{-6} \pm 7.77 \times 10^{-7}$ \\
\hline \multirow[t]{8}{*}{ TEAA } & glass & 100 & $1: 30$ & $5.32 \times 10^{-7} \pm 1.35 \times 10^{-7}$ \\
\hline & glass & 120 & $0: 30$ & $4.25 \times 10^{-7} \pm 6.76 \times 10^{-8}$ \\
\hline & PI & 100 & 2:00 & $4.10 \times 10^{-5} \pm 1.31 \times 10^{-6}$ \\
\hline & PI & 120 & $1: 30$ & $7.44 \times 10^{-7} \pm 6.81 \times 10^{-8}$ \\
\hline & PET & 100 & $2: 30$ & $3.01 \times 10^{-5} \pm 4.54 \times 10^{-6}$ \\
\hline & PET & 120 & 2:00 & $3.67 \times 10^{-6} \pm 4.66 \times 10^{-7}$ \\
\hline & paper & 100 & 2:00 & $4.00 \times 10^{-5} \pm 2.15 \times 10^{-5}$ \\
\hline & paper & 120 & 2:00 & $5.32 \times 10^{-6} \pm 3.06 \times 10^{-6}$ \\
\hline
\end{tabular}

${ }^{a}$ For comparison the resistivity of bulk $\mathrm{Al}$ is $2.65 \times 10^{-8} \Omega \mathrm{m}, 20^{\circ} \mathrm{C}$ (full data tables in Supporting Information).
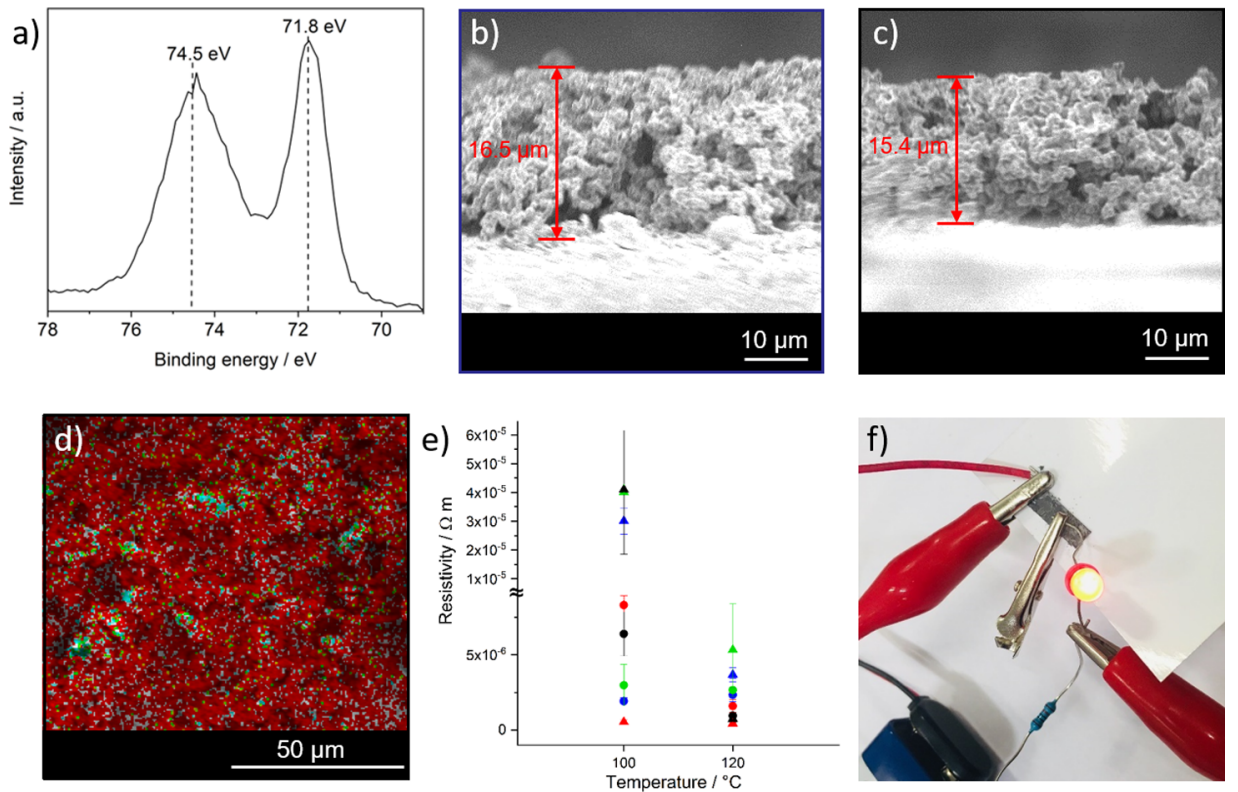

Figure 3. Properties of highly conductive Al films. (a) Al 2p XPS from a film deposited at $100{ }^{\circ} \mathrm{C}$ from DMEAA on paper showing surface aluminum oxide, $\mathrm{Al}_{2} \mathrm{O}_{3}$ at $75.4 \mathrm{eV}$ and $\mathrm{Al}$ metal at $71.8 \mathrm{eV}$. (b,c) Representative cross-sectional SEM images taken of Al films on PET and paper, respectively. (d) EDS analysis of the $\mathrm{Al}$ film deposited on paper showing good coverage of $\mathrm{Al}$ ( $\mathrm{Al}$, red regions) and some breakthrough to the underlying substrate (C, green regions). (e) Plot of resistivity vs temperature for depositions from DMEAA (circles) and TEAA (triangles) on glass (red), PI (green), PET (blue), and paper (black). (f) Example of an Al film deposited from DMEAA on paper at $100{ }^{\circ} \mathrm{C}$ cut to shape and used to light an LED in a circuit.

We have previously reported work on the effect of porous substrates such as paper, which is likely the cause for the differing morphology of films on paper reported herein. ${ }^{5}$ In these samples, the frequency of coalesced grains is high, with few spherical particles observed; this evidence of particles sintering together (sometimes referred to as necking) is supported by the excellent conductivity of these deposits (Table 1). Both DMEAA and TEAA have produced extremely low resistivity coatings at both 100 and $120{ }^{\circ} \mathrm{C}$. DMEAA produced the most consistent results, with resistivities in the range of $8.29 \times 10^{-6}$ to $9.57 \times 10^{-7} \Omega \mathrm{m}$, just one order of magnitude higher than bulk $\mathrm{Al}$-while all films display excellent conductivity; the best films were deposited at 120 ${ }^{\circ} \mathrm{C}$ on glass and PI. There is more variation in the resistivities reported from depositions from TEAA, as can be seen in Table 1 and also in the plot shown in Figure 3e. Measured resistivities range from $4.00 \times 10^{-5}$ to $4.25 \times 10^{-7} \Omega \mathrm{m}$ with the best coatings being deposited onto glass at 100 and $120{ }^{\circ} \mathrm{C}$ and on PI at $120{ }^{\circ} \mathrm{C}$; the depositions with the highest resistivities were being found on PI, PET, and paper at the lower decomposition temperature, $100{ }^{\circ} \mathrm{C}$-this information is consistent with the TGA data (Figure 1b), which shows complete decomposition of DMEAA at a lower temperature than for TEAA because TEA is slightly more basic than DMEA and so forms a stronger bonding interaction with the $\mathrm{Al}$ center which would require more energy to break. Sintering times vary between substrates because of a combination of their different thicknesses and thermal conductivities. To minimize 
these effects, substrates of similar thicknesses were chosen wherever possible, and substrates were preheated.

XPS measurements probing the $\mathrm{Al} 2 \mathrm{p}$ region were made at the surface of depositions and after a $300 \mathrm{~s} \mathrm{Ar}$ ion etch of all depositions (exemplar shown in Figure 3a). Two environments are present in each scan for every deposition, one each for aluminum oxide and the $\mathrm{Al}$ metal with no additional environments, which agrees with XPS data for the naturally oxidized $\mathrm{Al}$ metal foil. ${ }^{32}$ Aluminum oxide was observed in all surface scans at $c a .74 .5 \mathrm{eV}$, which agrees with literature values and is expected because XPS is a surface technique, with many metals (including $\mathrm{Al}$ ) forming passive oxide layers. ${ }^{32}$ Interestingly, $\mathrm{Al}$ metal is also present at the surface in a lower proportion at $c a .71 .7 \mathrm{eV}$, which is in agreement with the literature, revealing that not all the metal surface has been oxidized. After the $300 \mathrm{~s}$ etch, the ratios of oxide to the metal decrease ( 34.5 to $44.3 \% \mathrm{Al}$ metal), which is intuitive as more surface oxide has been removed by the etching process. Aluminum oxide is still present after $300 \mathrm{~s}$ etches, which is most likely due to the porous morphology of the films, allowing oxygen to penetrate forming a $3 \mathrm{D}$ network of surface alumina. A small shift in the binding energy of $\mathrm{Al}$ metal is observed (to $c a .71 .8 \mathrm{eV}$ ) but is within error limits. The nitrogen content is $0.0 \%$ in all films, which agrees with the theory that the volatile amines are completely removed, before the alane converts to $\mathrm{Al}$.

Cross-sectional SEM images were obtained for deposits on PI and PET (Figure 3b,c). Sufficient quality images for glass and paper could not be obtained because of issues involving sample preparation (scoring glass and cutting paper destroyed the film along the edge). Film thicknesses were estimated for PI and PET by taking three points along the film, with depositions on PET being marginally thicker (PI $=15.44 \mu \mathrm{m}$, PET $=16.46 \mu \mathrm{m})$.

EDS analysis of the Al coatings confirmed the presence of $\mathrm{Al}$, with very small amounts of $\mathrm{O}$ present $(<5.4 \%$ in all cases, table in SI). For example, when TEAA was used to deposit Al on paper and glass (at 120 and $100{ }^{\circ} \mathrm{C}$, respectively) EDS showed an $\mathrm{Al}$ content of $93.2 \%$ and $96.6 \%$, respectively. DMEAA liquid MOD inks used to deposit $\mathrm{Al}$ on PI and PET (at $120{ }^{\circ} \mathrm{C}$ ), for example, produced coatings with 85.1 and $78.5 \% \mathrm{Al}$ which can be attributed to varying levels of carbon detection. Carbon contamination varied the most between substrates, with PI and PET substrates containing the highest amount (11.4 and $16.1 \%$ respectively). This is most likely due to the electron beam breaking through the porous film to the carbon-based substrate, as the carbon contamination for the less porous film on paper is very small (3.4\%) and null for glass $(0.0 \%)$, which is not a carbon-based substrate. This theory is substantiated by the EDS-layered spectra (Figure 3d, full information in Supporting Information).

While all deposits of $\mathrm{Al}$ reported herein are highly conductive, depositions from DMEAA proved to be of much better quality, which adherent films being deposited on paper substrates at faster times, than the comparative depositions carried out at both 100 and $120{ }^{\circ} \mathrm{C}$ from TEAA (Table 1, Figure $3 \mathrm{e}$ ). As such, an example of an $\mathrm{Al}$ film deposited from DMEAA on paper at $100{ }^{\circ} \mathrm{C}$ was cut to shape and used to light an LED in a circuit highlighting the potential application for our methods (Figure 3f).

These hydride precursors have proven to be highly suitable for deposition of conductive Al, likely owning to the presence of reactive $\mathrm{Al}-\mathrm{H}$ bonds. The hydrido ligand acts threefold: first, the weak bond presents a low thermodynamic barrier to cross; second, each hydrogen in the $\mathrm{AlH}_{3}$ moiety provides an electron to reduce the (formally) $\mathrm{Al}(\mathrm{III})$ center to $\mathrm{Al}(0)$; and finally, because it is a gaseous byproduct, highly conductive features are afforded because contamination (typically carbon) is avoided in this entropically driven process.

\section{CONCLUSIONS}

In summary, the deposition of the liquid MOD inks DMEAA and TEAA has yielded the formation of highly conductive $\mathrm{Al}$ (mean electrical resistivity: $8 \times 10^{-6} \Omega \mathrm{m}$ ), approximately 300 times more resistive than bulk $\mathrm{Al}$ metal on glass, PI, PET, and paper. Such low resistivity has potential application in superior quality electronics, such as circuitry in high frequency devices. The use of thermal sintering at ultra-low temperatures (100 and $120{ }^{\circ} \mathrm{C}$ ) has shown to be an effective conversion tool which removes the need for multistep deposition routes or requirements for any catalyst additive. In addition, in our approach the careful selection and synthesis of MOD precursors that are liquids at room temperature has removed the need for dilution or further ink formulation, and as a consequence, this strategy has much potential in the realization of flexible and paper-based printing of highly conductive $\mathrm{Al}$ for use in electronic devices. Future work in this field should focus on the better precursor DMEAA because it exhibits superior performance. The precursor should be applied to more advanced/large-scaled techniques such as spin-coating or doctor-blading under inert conditions, prior to optimization eventually yielding intricate patterns via inkjet printing, from which scale up and inclusion within electronic circuitry would be an obvious next step.

\section{ASSOCIATED CONTENT}

\section{Supporting Information}

The Supporting Information is available free of charge at https://pubs.acs.org/doi/10.1021/acsami.0c05429.

${ }^{1} \mathrm{H}$ and ${ }^{13} \mathrm{C}$ NMR data of DMEAA. ${ }^{1} \mathrm{H}$ and ${ }^{13} \mathrm{C}$ NMR data of TEAA. Hypothetical decomposition schemes of (a) DMEAA an (b) TEAA, Background XRD patterns of substrates. Full (unnormalized) XRD data. Grain size analysis for top-down SEMs. Representative EDSlayered spectra of top-down SEMs, showing respective EDS breakdown. XPS survey spectra showing the Al 2s and $2 p$ transitions. Tabulated data for all depositions. Resistivity calculations (PDF)

\section{AUTHOR INFORMATION}

\section{Corresponding Author}

Caroline E. Knapp - Department of Chemistry, University College London, London WC1H OAJ, U.K.; ๑) orcid.org/00000003-4219-9313; Email: caroline.knapp@ucl.ac.uk

\section{Author}

Samuel P. Douglas - Department of Chemistry, University College London, London WC1H OAJ, U.K.; (1) orcid.org/ 0000-0003-4377-8851

Complete contact information is available at: https://pubs.acs.org/10.1021/acsami.0c05429 


\section{Author Contributions}

The manuscript was written through contributions of all authors. All authors have given approval to the final version of the manuscript.

\section{Notes}

The authors declare no competing financial interest.

\section{ACKNOWLEDGMENTS}

The authors thank the EPSRC for funding, S.P.D. (EP/ N509577/1). Dr. Sanjay Sathasivam for running the XPS.

\section{REFERENCES}

(1) Knapp, C. E.; Pugh, D.; McMillan, P. F.; Parkin, I. P.; Carmalt, C. J. Synthetic and Structural Studies of Donor-Functionalized Alkoxy Derivatives of Gallium. Inorg. Chem. 2011, 50, 9491-9498.

(2) Choi, Y.; Seong, K. d.; Piao, Y. Metal-Organic Decomposition Ink for Printed Electronics. Adv. Mater. Interfaces 2019, 6, 1901002.

(3) Seong, K.-d.; Kim, J. M.; Kang, J.; Hwang, M.; Lee, C.; Piao, Y. An Ultradurable and Uniform $\mathrm{Cu}$ Electrode by Blending Carbon Nanotube Fillers in Copper-Based Metal-Organic Decomposition Ink for Flexible Printed Electronics. Adv. Mater. Interfaces 2018, 5, 1800502.

(4) Zope, K. R.; Cormier, D.; Williams, S. A. Reactive Silver Oxalate Ink Composition with Enhanced Curing Conditions for Flexible Substrates. ACS Appl. Mater. Interfaces 2018, 10, 3830-3837.

(5) Knapp, C. E.; Chemin, J.-B.; Douglas, S. P.; Ondo, D. A.; Guillot, J.; Choquet, P.; Boscher, N. D. Room-Temperature Plasma-Assisted Inkjet Printing of Highly Conductive Silver on Paper. Adv. Mater. Technol. 2018, 3, 1700326.

(6) Knapp, C. E.; Metcalf, E. A.; Mrig, S.; Sanchez-Perez, C.; Douglas, S. P.; Choquet, P.; Boscher, N. D. Precursors for Atmospheric Plasma-Enhanced Sintering: Low-Temperature Inkjet Printing of Conductive Copper. ChemistryOpen 2018, 7, 850-857.

(7) Farraj, Y.; Grouchko, M.; Magdassi, S. Self-Reduction of a Copper Complex MOD Ink for Inkjet Printing Conductive Patterns on Plastics. Chem. Commun. 2015, 51, 1587-1590.

(8) Lee, Y.-I.; Choa, Y.-H. Adhesion Enhancement of Ink-Jet Printed Conductive Copper Patterns on a Flexible Substrate. J. Mater. Chem. 2012, 22, 12517-12522.

(9) Choi, Y.-H.; Hong, S.-H. Effect of the Amine Concentration on Phase Evolution and Densification in Printed Films Using $\mathrm{Cu}$ (II) Complex Ink. Langmuir 2015, 31, 8101-8110.

(10) Lee, H. M.; Choi, S.-Y.; Kim, K. T.; Yun, J.-Y.; Jung, D. S.; Park, S. B.; Park, J. A Novel Solution-Stamping Process for Preparation of a Highly Conductive Aluminum Thin Film. Adv. Mater. 2011, 23, $5524-5528$.

(11) Bruno, T. J.; Lide, D. R.; Haynes, W. M. CRC Handbook of Chemistry and Physics: A Ready-Reference Book of Chemical and Physical Data; Haynes, W. M., Lide, D. R., Bruno, T. J.; CRC Press, 2017.

(12) Downs, A. J. Chemistry of Aluminium, Gallium, Indium and Thallium; Blackie Academic \& Professional, 1993; p 526.

(13) Lee, H. M.; Choi, S.-Y.; Jung, A.; Ko, S. H. Highly Conductive Aluminum Textile and Paper for Flexible and Wearable Electronics. Angew. Chem. Int. Ed. 2013, 52, 7718-7723.

(14) Wee, A. T. S.; Murrell, A. J.; Singh, N. K.; O’Hare, D. M.; Foord, J. S. Surface Decomposition Mechanism of the Novel Precursor Bistrimethylamine Aluminium Hydride on GaAs (100). Vacuum 1990, 41, 968-971.

(15) Simmonds, M. G.; Gladfelter, W. L. Chemical Vapor Deposition of Aluminum. The Chemistry of Metal CVD; Wiley, 2007; pp 45-103.

(16) Knapp, C. E.; Marchand, P.; Dyer, C.; Parkin, I. P.; Carmalt, C. J. Synthesis and Characterisation of Novel Aluminium and Gallium Precursors for Chemical Vapour Deposition. New J. Chem. 2015, 39, 6585-6592.
(17) Gladfelter, W. L.; Boyd, D. C.; Jensen, K. F. Trimethylamine Complexes of Alane as Precursors for the Low-Pressure Chemical Vapor Deposition of Aluminum. Chem. Mater. 1989, 1, 339-343.

(18) Humphries, T. D.; Munroe, K. T.; Dewinter, T. M.; Jensen, C. M.; Mcgrady, G. S. NMR Spectroscopic and Thermodynamic Studies of the Etherate and the $\alpha, \mathrm{A}^{\prime}$, and $\gamma$ Phases of $\mathrm{AlH}_{3}$. Int. J. Hydrogen Energy 2013, 38, 4577-4586.

(19) Eaborn, C. Hydrides of the Elements of Main Groups I-IV. J. Organomet. Chem. 1972, 35, C52.

(20) Rockenberger, J.; Zürcher, F.; Guo, W. Aluminum Inks and Methods of Making the Same, Methods for Depositing Aluminum Inks, and Films Formed by Printing and/or Depositing an Aluminum Ink. U.S. Patent 20,100,022,078 A1, January, 2010.

(21) Curtis, C. J.; Miedaner, A.; Van Hest, M. F. A. M.; Ginley, D. S. Printing Aluminum Films and Patterned Contacts Using Organometallic Precursor Inks. WO2009059273 A3, November 3, 2009.

(22) Lee, H. M.; Lee, H. B.; Jung, D. S.; Yun, J.-Y.; Ko, S. H.; Park, S. B. Solution Processed Aluminum Paper for Flexible Electronics. Langmuir 2012, 28, 13127-13135.

(23) Lee, H. M.; Choi, S.-Y.; Jung, A. Direct Deposition of Highly Conductive Aluminum Thin Film on Substrate by Solution-Dipping Process. ACS Appl. Mater. Interfaces 2013, 5, 4581-4585.

(24) Jung, S.-h.; Choi, D. Y.; Lee, H. M. Roll-to-Roll Processed, Highly Conductive, and Flexible Aluminum (Al) Electrodes Based on Al Precursor Inks. RSC Adv. 2018, 8, 19950-19957.

(25) Lee, H. M.; Seo, J. Y.; Jung, A.; Choi, S.-Y.; Ko, S. H.; Jo, J.; Park, S. B.; Park, D. Long-Term Sustainable Aluminum Precursor Solution for Highly Conductive Thin Films on Rigid and Flexible Substrates. ACS Appl. Mater. Interfaces 2014, 6, 15480-15487.

(26) Shen, Z.; Matsuki, Y.; Shimoda, T. Selected Deposition of High-Quality Aluminum Film by Liquid Process. J. Am. Chem. Soc. 2012, 134, 8034-8037.

(27) Garner, W. E.; Haycock, E. W. The Thermal Decomposition of Lithium Aluminium Hydride. Proc. R. Soc. London, Ser. A 1952, 211, $335-351$

(28) Humphries, T. D.; Munroe, K. T.; Decken, A.; McGrady, G. S. Lewis Base Complexes of $\mathrm{AlH}_{3}$ : Prediction of Preferred Structure and Stoichiometry. Dalton Trans. 2013, 42, 6965-6978.

(29) Humphries, T. D.; Munroe, K. T.; Decken, A.; McGrady, G. S. Lewis Base Complexes of $\mathrm{AlH}_{3}$ : Structural Determination of Monomeric and Polymeric Adducts by X-Ray Crystallography and DFT Calculations. Dalton Trans. 2013, 42, 6953-6964.

(30) Kamyshny, A.; Magdassi, S. Conductive Nanomaterials for 2D and 3D Printed Flexible Electronics. Chem. Soc. Rev. 2019, 48, 17121740.

(31) Klingner, S.; Voigts, F.; Viöl, W.; Maus-Friedrichs, W. Analysis of Plasma Degreased Aluminium Foil with XPS. Surf. Eng. 2013, 29, 396.

(32) Strohmeier, B. R. Characterization of an Activated Alumina Claus Catalyst by XPS. Surface Science Spectra 1994, 3, 141-146. 